ÉGYPTE monde arabe

\section{Égypte/Monde arabe}

15-16 | 1993

Les crises soudanaises des années 80

\title{
Traditionnalisme et modernisation : une perspective politique
}

\section{Mohammed Beshir Hamid}

\section{(2) OpenEdition \\ 1 Journals}

\section{Édition électronique}

URL : https://journals.openedition.org/ema/1099

DOI : 10.4000/ema.1099

ISSN : 2090-7273

\section{Éditeur}

CEDEJ - Centre d'études et de documentation économiques juridiques et sociales

\section{Édition imprimée}

Date de publication : 31 décembre 1993

Pagination : 207-226

ISSN : 1110-5097

\section{Référence électronique}

Mohammed Beshir Hamid, «Traditionnalisme et modernisation : une perspective politique », Égypte/ Monde arabe [En ligne], 15-16 | 1993, mis en ligne le 08 juillet 2008, consulté le 07 juillet 2022. URL : http://journals.openedition.org/ema/1099; DOI : https://doi.org/10.4000/ema.1099

Ce document a été généré automatiquement le 7 juillet 2022.

Tous droits réservés 


\title{
Traditionnalisme et modernisation : une perspective politique
}

\author{
Mohammed Beshir Hamid
}

\section{NOTE DE L'ÉDITEUR}

Traduit de l'anglais par Samia Rizq.

1 A cheval sur des frontières stratégiques et culturelles, le Soudan, de par sa position géographique, est au croisement de groupes ethniques et linguistiques qui sont à l'origine de son identité mais aussi de multiples conflits. Depuis son indépendance, le Soudan se caractérise, sur le plan politique, par une alternance de régimes civils et militaires. Sous les premiers, ce sont des partis traditionalistes qui gouvernent une démocratie parlementaire corrompue, quasi anarchique, alors que les derniers présentent, dans la plupart des cas, tous les traits caractéristiques des régimes conservateurs et des dictatures. Dans cet étrange modèle, qui se reproduit avec une périodicité assez régulière, les éléments modernistes servent de catalyseur lors du passage d'un cycle à l'autre sans assumer, ni dans l'un ni dans l'autre, un rôle important ou durable.

2 Le pays se trouve ainsi enfermé dans un cercle vicieux de contradictions et de confrontations allant au-delà de la ligne de démarcation habituelle entre traditionalisme et modernisme. L'émergence du courant islamiste, qui rejette tout à la fois le «sectarisme» du parti traditionnel et le «sécularisme» des éléments modernistes, constitue essentiellement une sorte de «traditionalisme radical». De même, l'émergence, au sud du Soudan, de l'insurrection armée dont l'objectif déclaré est la restructuration des relations de pouvoir sur des bases socialistes et laïques, peut apparaître comme une nouvelle forme de modernisme fondé essentiellement, mais pas exclusivement, sur l'appartenance tribale.

3 Cette étude se propose d'examiner l'interaction de ces forces divergentes, concurrentes et contradictoires. Tout d'abord, nous étudierons les problèmes que posent la définition 
du modernisme et la possibilité d'appliquer le modèle occidental aux sociétés africaines. Ensuite, nous traiterons de l'évolution, de la nature et du rôle des partis dans le processus politique. Nous analyserons alors les cycles civils/militaires de l'histoire politique du Soudan depuis son indépendance. Enfin, dans la quatrième partie, nous exposerons les revendications propres à chacun des deux courants, islamiste et séculier.

Un problème de définition

4 Sur le plan conceptuel, le terme de "tradition » sert à " combler un vide théorique considérable; il réfère évidemment à la vision conservatrice répandue qui consiste à énoncer que, surtout dans le domaine de l'histoire, il est dangereux d'avancer des théories et que celles-ci peuvent même, dans l'exercice de la politique, être dénuées de sens $^{1}$. Les traditions peuvent relever à la fois du social et du politique mais certaines d'entre elles peuvent aussi, selon le cas, conduire à des acceptions différentes du mot «tradition ». Ce terme est essentiellement évoqué pour affirmer le respect du passé et l'idée actuelle selon laquelle le présent doit être interprété non pas comme «imitation» d'une réalité sociale et politique, mais seulement comme une " continuation» de cette réalité. La tradition concerne donc, immanquablement, l'attachement à un passé et, par conséquent, à une politique et à une organisation sociale et institutionnelle qui « rendent présent ce passé $»^{2}$.

5 La tradition renferme des éléments fondamentaux qui poussent à une certaine ardeur dans les «actions délibérées » ainsi que dans les pensées, croyances, perceptions et associations qui poussent à les accomplir. Les sentiments suscités par la tradition sont forcément des sentiments partagés (la tradition «solitaire» n'existe pas) et le comportement traditionaliste est évidemment, en partie, motivé par le fait qu'il est partagé. Ainsi, au-delà de leur valeur individuelle, ces pratiques ont une valeur sociale et l'individu qui s'y conforme jouit d'une reconnaissance collective. De plus, en tendant à créer un ensemble de "représentations partagées » et en permettant de distinguer, du moins en partie, le caractère moral et individuel de ceux qui s'opposent à cet ensemble, les traditions constituent, dans un sens, un élément moderniste ${ }^{3}$.

6 Le traditionalisme, c'est donc toute doctrine ou toute politique fondée sur la défense de la tradition. Par contre, un des problèmes que l'on rencontre lorsqu'on tente de définir la modernisation, c'est que ce terme est utilisé dans des contextes différents, par différentes disciplines avec, à chaque fois, une approche et une acception particulière. Pour la sociologie, par laquelle ce terme a été utilisé en premier, la modernisation c'est le changement des valeurs, de la structure et de la formation de "l'homme moderne». En économie, le mot « modernisation » sert à déterminer le degré de développement ou de croissance de différentes grandeurs dites "économiques». Dans le domaine sociopolitique, les approches sont variables. Selon Apter, «l'aspect dynamique de la modernisation dans le cadre des études politiques pourrait se définir, de manière générale, comme étant le processus de relations humaines de plus en plus complexes par lequel toute action politique doit s'exercer. D'où les sérieux problèmes politiques qui en découlent. La politique est devenue, dans une large mesure, l'art de se distinguer tout en s'intégrant dans les structures organisationnelles. L'action politique née d'une telle complexité n'est cependant pas l'œuvre des leaders politiques en dehors de tout contexte. $»^{4}$

7 En science politique, cette approche revêt une connotation qui provient essentiellement, mais non exclusivement, d'une forte tradition de la théorie sociale et 
politique selon laquelle cette notion est basée sur des valeurs occidentales. Lerner, par exemple, se sert du modèle occidental de modernisation comme point de départ, plus peut-être par nécessité que par choix (parce que, dit-il, c'est ce modèle qui «s'impose à nous »). Il préfère cependant utiliser le terme de «modernisation» plutôt que celui d'«occidentalisation» à cause des sentiments d'hostilité anti-occidentale que l'on trouve, dans les sociétés du tiers-monde, parmi ceux qui prônent cette modernisation ${ }^{5}$.

8 Ainsi, pour Lerner, la modernisation est un processus social dont le développement, à la manière occidentale, dépend de la composante économique. Comme Lerner, Deutsch considère que le processus de "mobilité sociale" ouvre la voie aux processus de "construction de la nation", étroitement liés à un modèle "occidental» de développement. Formulant une variante de la théorie de la modernisation, Shils avance une théorie des "élites » selon laquelle " développement » et «modernisation » sont essentiellement une forme d'« occidentalisation». Avec sa volonté de modernité, l'élite a pour rôle de créer les mécanismes de l'intégration politique et les stratégies particulières constituant le cadre et les instruments nécessaires au processus de modernisation.

9 Le passage de la société traditionnelle à la société "participante " (c'est-à-dire moderne) implique un stade intermédiaire que Lerner appelle "transitoire», phase ambivalente, indépendante aussi bien du passé que du présent, et pourtant en rapport avec l'un et l'autre. Les composantes dynamiques de cette phase transitoire se mesurent, selon Lerner, aux indices d'urbanisation, d'alphabétisation, d'équipement en appareils radio-télévisuels et de participation, phénomènes qui lient le «changement institutionnel' au «mode de vie personnel $»^{6}$. Ainsi, la transformation des conditions sociales, surtout dans le domaine de l'éducation et des communications, tend à augmenter la capacité d'un système à mettre en œuvre des politiques modernes complexes répondant au plus grand nombre d'intérêts différents.

Bien qu'ils puissent constituer les principales variables d'analyse du processus de transition, les "indices» de Lerner ne permettent pas pour autant d'identifier le rapport qui existe entre forces traditionnelles et forces modernistes, ni d'expliquer certains aspects sociaux du comportement traditionaliste face à la modernisation. Présumer que l'éducation est liée à la modernisation suppose, comme le dit Bechtold, que «le comportement traditionnel est irrationnel alors que, dans certaines circonstances, il semble aussi probable que l'adhésion aux valeurs traditionnelles puisse constituer, même pour une personne instruite, une décision rationnelle $»^{7}$.

11 La manière de définir la modernisation par opposition à la tradition en termes dichotomiques et de ne considérer que l'aspect négatif de cette dernière est en rapport avec ce qui précède. Une telle méthode ne tient pas compte des différences existant entre les sociétés empiriques classées sous la catégorie résiduelle de tradition ${ }^{8}$. En qualifiant les sociétés modernes de "participatives", Lerner soulève la question de savoir si la tradition qui consiste, dans les sociétés tribales d'Afrique, à participer à la vie communautaire dans une sorte de socialisme primitif, ne peut être considérée comme une participation simplement parce qu'elle n'est pas conforme à la modernité selon la tradition occidentale. En effet, la tendance à confondre "modernité » et " occidentalisme" et à considérer les deux termes comme des synonymes pose le délicat problème de l'ethnocentrisme. Soutenir, comme le fait Lerner que le modèle "occidental» ne l'est que du point de vue historique et que, du point de vue sociologique, il s'agit d'un phénomène global, c'est vouloir appliquer aux pays en 
développement des «modèles-type ». L'application universelle de certains critères relève alors d'une démarche ethnocentrée qui prétend prévoir un devenir unique des peuples.

12 Ainsi, la modernisation n'est autre qu'une voie menant à un type particulier de développement industriel-capitaliste lié à une forme de régime politique basé sur la conception occidentale de « démocratie libérale ». Cela sous-entend que, tant au niveau économique que politique, tout écart par rapport à la «norme occidentale » est considéré comme un échec dans le processus de modernisation (Eisenstadt), tandis que l'adoption d'une voie différente, tel que le communisme par exemple, est rejetée comme un "mal de la transition" (Rostow). En Afrique et dans le Monde arabe, le processus de modernisation est en fait plus complexe. Les conceptions et les choix, qu'ils soient politiques ou économiques, s'expliquent mieux en termes d'exigence développementaliste ayant engendré un ensemble d'institutions autoritaires. La dynamique de la construction identitaire agissant à de multiples niveaux et dans des directions différentes, l'expérience locale se trouve alors influencée par des idéologies diverses et concurrentes. Le processus identitaire se traduit d'habitude par «le rejet des valeurs et de la culture occidentales joint à l'affirmation des racines et des valeurs précoloniales (ex. islamisme, arabité, négritude); l'adaptation des idéologies européennes aux conditions et besoins locaux des autochtones (ex. socialisme arabe, socialisme africain) ; le choix de valeurs et d'idéologies universellement reconnues (ex. marxisme, communisme de type soviétique ou chinois) »".

13 Les processus de démocratisation actuellement en cours en Afrique ont ajouté un élément nouveau à la situation africaine; ces processus répondent à une demande de pluralisme que les régimes à parti unique rejettent; l'instauration du pluralisme signifie, pour (es régimes à tendance socialiste, la perte d'une part de leur légitimité et de leur viabilité. Toutefois, ceci ne doit pas nécessairement laisser entendre que le modèle capitaliste de développement fondé sur l'économie de marché soit mieux accueilli. Bon nombre d'intellectuels africains expriment leurs doutes quant aux intentions cachées de l'Occident qui, pour accorder une aide financière, pose maintenant comme condition la démocratisation du pays concerné. Les exigences relatives à l'ajustement structurel semblent incompatibles avec le souci de démocratisation dans la mesure où elles ne peuvent être appliquées que par des régimes autoritaires et non par des régimes démocratiques. Certains mouvements prodémocratiques en Afrique ont évidemment commencé à se soulever contre les mesures d'austérité économique imposées par les donateurs étrangers.

Dans des sociétés traditionnelles comme le Soudan, le problème n'est pas le changement en soi, mais plutôt la détermination des facteurs de changement qu'impose la modernisation ou l'accélération du processus de modernisation ${ }^{10}$.

Les partis politiques

15 L'histoire moderne du Soudan en tant qu'entité politique commence au début du XIX siècle avec la pénétration de l'Égypte et de l'Europe. L'occupation turco-égyptienne de 1821 à 1885 suscite la résistance soudanaise et inspire finalement un renouveau islamique nationaliste sous le leadership de Muhammad Ahmad al-Mahdi, renouveau qui culmine avec la reconstruction d'un Soudan politiquement indépendant.

16 Alors que le mahdisme, en tant que sentiment religieux et mouvement politique, devait jouer un rôle important dans le développement de la politique soudanaise, le mahdisme en tant qu'État n'a pas fait long feu. Khalifa Abdullahi, le successeur d'al-Mahdi, est 
vaincu en 1898 par les forces alliées de la Grande-Bretagne et de l'Égypte. Le pays est soumis à un condominium égypto-britannique - une innovation en matière de droit international. L'Égypte étant elle-même sous protectorat britannique, le vrai contrôle du Soudan est en fait aux mains des Anglais. «La nature politique et administrative de ce double régime devait influencer l'évolution et les orientations de la marche du Soudan vers l'indépendance. Les adeptes du mahdisme, les Ansar, ont été de plus en plus assimilés au mouvement pour l'indépendance du Soudan qui, en union avec la Grande-Bretagne, tentait de mettre fin à l'influence de l'Égypte. De son côté, la confrérie religieuse rivale, la Khatmiyya, s'unissait à l'Égypte sous le slogan de l'unité de la vallée du Nil pour se libérer de la domination britannique. Les principaux partis politiques qui se sont formés par la suite ont suivi la même répartition: le parti alUmma représentait les Ansar et le parti Achiqa (appelé plus tard Parti de l'union nationale, PUN) était appuyé par la Khatmiyya. $»^{11}$

Des changements de camp se sont produits aussi bien au sein d'un même parti que d'un parti à l'autre. Ces changements n'étaient pas le fait de déceptions par rapport à la politique de tel ou tel groupe mais plutôt des choix individuels entre des voies différentes visant toutes un objectif nationaliste unique. Dans les partis traditionalistes, la prise de décision était hautement centralisée avec très peu de participation politique ou de voies ouvertes à l'expression de la base. Cependant, dominés par l'esprit de (action, les partis étaient incapables de sauvegarder leur cohésion interne. La politique de faction est vite devenue la caractéristique de l'après indépendance et a contribué de manière décisive à l'échec de la première expérience parlementaire au Soudan.

Outre les deux partis traditionalistes, il existait d'autres partis qui ont choisi de rester loin des politiques confrériques sans s'abstenir pour autant de conclure des alliances lactiques avec l'un ou l'autre des deux grands partis, lorsqu'il y allait de leur intérêt. Le Parti communiste soudanais (PCS) tire ses origines, en tant qu'organisation politique officielle, du Mouvement soudanais de libération nationale (MSLN), formé en 1946 et issu du mouvement communiste égyptien. Durant la période qui a précédé l'indépendance, la direction du MSLN a déployé de gros efforts pour attirer, sous la bannière de la lutte anti-colonialiste, les ouvriers, les paysans et les étudiants. Durant la période de l'après indépendance, le PCS a réussi à exercer son influence et à se faire appuyer par ces groupes. Avec sa discipline et son expérience des activités clandestines, il est devenu un des partis communistes d'Afrique et du Monde arabe les mieux organisés. Le communisme a surtout attiré des groupes qui avaient rejeté l'affiliation confrérique ou dont la fidélité aux factions avait suffisamment faibli pour que le PCS devienne pour eux une alternative attrayante. Cependant, «il est important de noter que chaque fois que la question du communisme et de sa légalité était soulevée en public, elle était abordée sous l'angle du communisme vis-à-vis de l'islam et non pas sous celui de l'idéologie communiste sous ses aspects politique, économique ou social $»^{12}$.

19 Pleinement conscient de ce fait, le PCS a dû, dans la plupart des cas, reconnaître la nécessité de coopérer avec les partis issus des confréries pour certaines questions politiques ou dans certains programmes. Ces contraintes mettaient le PCS face à un dilemme ; à travers cette coopération ou cette confrontation, il avait peu de chances de rompre le bastion confrérique, et cependant sa survie dépendait de l'affaiblissement des fidélités confrériques sous l'impact économique, social et culturel de la modernisation ${ }^{13}$. Il n'est donc pas étonnant que la flexibilité idéologique et les besoins 
contradictoires d'arriver à un compromis politique aient abouti, au sein du PCS, au même factionnalisme (exprimé en termes de "révisionnisme » idéologique) que celui qui sévissait dans les partis à base confrérique.

L'islam populaire militant des partis confrériques s'est vu concurrencé et défié par l'émergence du mouvement islamiste radical dirigé par le Parti des Frères musulmans qui, comme le Parti communiste, était issu d'une organisation militante religieuse et politique ayant vu le jour en Égypte en 1928. Au Soudan, c'est en 1964 que ce mouvement acquit une importance nationale et une reconnaissance politique, lorsqu'il s'organisa en tant que front islamique constitutionnel (FIC). Encore une fois, comme les communistes, le FIC était assimilé à l'élite instruite. Les deux groupes sollicitèrent les mêmes strates sociales, surtout celles qui avaient été déçues par les échecs des régimes fondés sur le confrérisme.

Grâce à leur politique "agressive ", les Frères musulmans ont bénéficié d'un appui régulier ; leur militantisme leur a fait gagner plus de voix et d'influence que ne l'aurait permis une simple adhésion à leur association. Selon un observateur de la scène soudanaise, l'idéologie consistant à prôner l'établissement d'un État et d'une société fondés sur les préceptes de l'islam est essentiellement "fondamentaliste»; cette idéologie doit être considérée surtout comme un développement du soufisme et du mahdisme, jusqu'alors très influents au Soudan.

En effet, ce développement peut être considéré comme une réponse à l'urbanisation et aux autres formes de changement social qui ont contribué à affaiblir les groupes les plus anciens et continueront dans l'avenir à le faire, du moins de l'avis des Frères musulmans ${ }^{14}$.

L'hostilité partagée des Frères musulmans et des communistes à l'égard des partis traditionalistes n'était dépassée que par la profonde animosité qui opposait ces deux partis radicaux. Bien qu'il ait existé entre eux certaines similitudes, de par leur origine, leurs intérêts, leur mode d'organisation et leurs engagements, il reste que leurs orientations et leurs projets politiques divergeaient. Chacun reconnaissait ne pouvoir atteindre ses objectifs que par l'élimination de l'autre.

Il existait également des partis régionaux à l'ouest, à l'est et au sud du pays. Leur impact sur la politique soudanaise a cependant été marginal. Plus significatifs et d'une influence plus directe ont été les mouvements rebelles qui sont apparus au sud du Soudan : l'Anya Nya et l'actuel Mouvement de libération des peuples du Soudan (MLPS), branche politique de l'Armée de libération des peuples du Soudan (ALPS) et formé en 1983.

25 Le mouvement Anya Nya a combattu le gouvernement soudanais pendant plus de dix ans, jusqu'à la convention d'Addis Abeba en 1972, qui accorda au Sud un gouvernement régional. Quant au MLPS/ALPS, il entra en rébellion en 1983 et, contrairement au mouvement Anya Nya, il se déclara mouvement nationaliste luttant pour la restructuration des rapports de pouvoir au Soudan.

Les deux mouvements ont, à tour de rôle, exercé une influence profonde sur l'alternance des cycles civils/militaires qui se sont succédé au Soudan.

Les cycles civils et militaires

27 Ironie $\mathrm{du}$ sort: après une transition sans heurts, la déclaration officielle d'indépendance, en janvier 1956, arrive dans le sillage d'une révolte sudiste qui aura de sérieuses répercussions sur les relations nord-sud. Toutes les attentes, toutes les 
espérances sont presque immédiatement sapées par l'intensification des rivalités entre les partis traditionalistes et par les clivages nés de leurs dissensions. En 1956, la Khatmiyya se sépare du PUN et forme un nouveau parti,' le Parti démocrate populaire (PDP), démarche précipitée par la décision prise par Isma'il al-Azhari, chef du PUN, d'opter (sous la forte pression politique exercée par le Parti al-Umma) pour l'entière indépendance du Soudan et non pour l'union avec l'Égypte. Le PDP forme ensuite avec le Parti al-Umma, malgré leurs rapports tendus, un gouvernement de coalition.

Les élections de 1958 allaient ramener au pouvoir cette même coalition Umma/PDP, qui était déjà en voie de désintégration du fait des divergences les opposant, surtout en ce, qui concerne l'aide américaine. Le système parlementaire copié sur le modèle de Westminster fut un échec, car le contexte politique du Soudan ne pouvait, ni au niveau national ni au niveau local, convenir à un tel système. La politique factionnelle de l'élite traditionnelle tendait à neutraliser et émousser les fonctions de participation et de représentation politiques. «L'échec le plus flagrant se produisit dans le Sud où, par négligence ou par manipulation, l'insensibilité aux injustices et aux aspirations régionales avait inexorablement créé une situation explosive. $»^{15}$ La décision prise par le Premier ministre, membre d'al-Umma, de faire appel à l'armée plutôt que de faire face à une probable défaite parlementaire et à une prise du pouvoir par un gouvernement de coalition PUN-PDP montre bien la fragilité du système politique et le degré d'animosité qui régnait entre les partis traditionalistes. Les militaires, qui avaient à leur tête le général Ibrahim Abboud, eurent l'obligeance d'accepter et, en novembre 1958, ils mirent fin à cette première expérience de gouvernement parlementaire au Soudan.

Le régime de Abboud ne s'est pas radicalement écarté de la politique traditionaliste ; il a su maintenir des liens avec les éléments influents de l'establishment qu'il venait de renverser. C'est ce qui, en lait, a constitué la base sociale du régime. Cependant, les rivalités au sein même de l'establishment étaient telles, que des liens étroits entretenus avec l'une des parties pouvaient avoir une influence inverse sur les rapports du régime avec les autres parties. C'est ainsi que certains milieux dirigeants se sont retrouvés, à certaines occasions, dans les rangs de l'opposition ${ }^{16}$.

31 Le régime militaire conservateur de Abboud a tenté, par divers moyens, de résoudre les problèmes politiques et économiques du Soudan. Devant l'échec de sa politique d'islamisation et d'arabisation forcée dans le sud, le régime a eu recours à un moyen simpliste et maladroit; il a fait appel à la force. Une escalade de la violence ne pouvait qu'aggraver une situation déjà périlleuse. Aux opérations militaires engagées dans le sud du pays par le gouvernement, la guérilla Anya Nya riposta en réclamant ouvertement la sécession.

Double échec pour le régime : sur le plan politique, il n'a pas réussi à résoudre les problèmes de gouvernement et de représentation; sur le plan militaire, il n'est pas parvenu à mettre un terme à la guerre civile. Au nord, l'opinion publique est de plus en plus mécontente, aussi bien de la politique trop sévère adoptée dans le sud du pays par le gouvernement, que de la corruption et de l'incompétence économique et politique de celui-ci. En octobre 1964, un soulèvement populaire unique renverse le pouvoir.

La révolution d'octobre 1964 est devenue un "héritage » en matière de politique soudanaise. Les groupes professionnels - universitaires, avocats, médecins, étudiants - et les syndicats qui ont renversé le régime militaire ont œuvré en marge du pouvoir traditionaliste établi et donc en opposition avec lui. Leur objectif était de mobiliser les 
travailleurs en milieu urbain et les paysans, et certains d'entre eux ont mené leur action au sein du PCS. Il n'est donc pas surprenant que le front des professionnels qui constituaient le nouveau gouvernement civil ait commencé à mener une campagne en faveur de programmes politiques appelant à un changement socio-économique radical. La réaction des partis traditionalistes a cependant été très rapide et a réussi à atténuer l'impact, sur le gouvernement de transition, des éléments les plus à gauche.

Avec les élections d'avril 1965, la coalition gouvernementale Umma-PUN qui était, de son propre aveu, un gouvernement conservateur, revient au pouvoir. La réapparition des partis politiques ressuscite et aggrave les vieilles divergences factionnelles et idéologiques régionales. Les partis politiques n'ont ni le désir ni le pouvoir de faire face aux problèmes complexes auxquels le pays est confronté.

C'est dans un contexte de crises politiques et constitutionnelles récurrentes qu'une série de coalitions peu viables se succèdent entre juin 1965 et mai 1969. L'optimisme de l'après-révolution cède la place à un désenchantement général vis-à-vis du système politique, et la situation s'aggrave en raison de la détérioration des conditions économiques et de l'intensification de la guerre civile au sud du Soudan ${ }^{17}$.

En mai 1969, le coup d'État monté par de jeunes officiers armés sous le commandement $\mathrm{du}$ colonel Ja'far Muhammad Nimeyri met fin à la deuxième phase du régime parlementaire. Le nouveau régime ne se présente pas comme issu d'un coup d'État militaire, mais comme le prolongement de la révolution d'octobre, révolution qu'il accuse les partis traditionalistes d'avoir trahie. Quelques éléments de la gauche et quelques nationalistes nassériens vont $s^{\prime} y$ identifier et chercher à exercer leur influence sur l'engagement idéologique des jeunes militaires, en les orientant dans le sens d'une révolution socialiste qui transformerait la société soudanaise.

Le régime radical réussit à écraser toute résistance, toute opposition des groupes traditionalistes et en particulier des Ansar. L'alliance avec la gauche cependant ne fera pas long feu: vers 1970, des divergences sur l'orientation panarabe du régime commencent à surgir. La direction du PCS est partagée quant à l'appui à apporter au pouvoir en place : les uns, en raison des tendances «socialistes » des jeunes officiers, sont favorables à la collaboration; les autres, y compris le secrétaire général 'Abd alKhaliq Mahjub, sont plus sceptiques quant aux ambitions idéologiques du régime. Ces dissensions vont entrâner la révocation des communistes et des éléments de gauche et le régime entamera une politique anti-communiste : à la suite de la tentative de coup d'État inspirée par les communistes en juillet 1971, quatorze leaders du PCS, y compris Mahjub, sont exécutés et une épuration massive des communistes a feu.

Après s'être aliéné aussi bien la gauche que la droite, Nimeyri va rapidement consolider sa position en essayant d'institutionnaliser et de légitimer son système politique. En octobre 1971, il est élu par plébiscite président de la République. Il s'empresse alors de trouver une solution politique au problème du Sud. La convention d'Addis Abeba, en mars 1972, met fin à la guerre civile et accorde aux provinces du Sud une autonomie régionale. Une nouvelle constitution reconnaît l'Union socialiste soudanaise (USS) comme seule organisation politique $d u$ pays. Par une série de décrets de "dénationalisation, des institutions économiques créées au début de la phase de radicalisme se trouvent démantelées par le régime. Une nouvelle politique d'ouverture (infitah) destinée à attirer les investissements occidentaux et arabes est lancée par le gouvernement ${ }^{18}$. 
39 La rupture avec la gauche et la réévaluation de la politique économique qui s'ensuivit ne laissaient prévoir pour autant aucun rapprochement immédiat avec les dirigeants des partis traditionnels. Les leaders des Partis al-Umma, DUP et Frères musulmans opposition en exil - ont constitué, à Londres, un Front national dont les tentatives de coup d'État ont failli renverser Nimeyri en septembre 1975 et en juillet 1976. Cependant, en juillet 1977, deux leaders du Front national, Sadiq al-Mahdi, du parti alUmma, et Hasan al-Turabi, des Frères musulmans, décident d'adopter une politique de réconciliation avec le régime Nimeyri. Les raisons en sont multiples : pour Nimeyri, il s'agit d'éviter une recrudescence des confrontations sanglantes, alors qu'al-Mahdi et Turabi, apparemment incapables de le déloger par la force, cherchent à opérer les changements qu'ils souhaitent en agissant de l'intérieur. Comme l'a si bien noté un auteur de l'époque, « une telle action a dû être facilitée par le fait que, Nimeyri étant revenu sur ses tendances radicales du début, les clivages idéologiques entre les deux parties se sont estompés. L'impact de facteurs connexes internes, externes et économiques tend à créer un ensemble de circonstances plus favorables qui se prêtent bien, pour les uns 'et pour les autres, à l'idée d'une réconciliation nationale. $»^{19}$

40 Les espérances de Sadiq al-Mahdi concernant les changements substantiels et une participation aux principales affaires politiques sont cependant vite démenties. II met fin à sa coopération avec le régime en s'abstenant toutefois de jouer un rôle dans l'opposition. De leur côté, par leur collaboration active, les Frères musulmans sont devenus la forcé motrice orientant le régime vers l'islamisation. En septembre 1983, Nimeyri décrète l'application de la charî'a et considère cette mesure comme une « révolution juridique». A ce moment, Sadiq al-Mahdi est détenu pour avoir critiqué publiquement les mesures adoptées par Nimeyri à ce sujet, mesures qu'il qualifie d'essentiellement non islamiques. Paradoxalement, la mise en œuvre de l'islamisation semble ne pas satisfaire les attentes politiques des Frères musulmans, qui se sont pourtant donné tant de peine pour y parvenir. A peine Nimeyri a-t-il obtenu leur appui politique grâce au processus de réconciliation qu'il essaie, en décrétant l'islamisation, de les attirer sur une plate-forme idéologique commune pour se retourner ensuite contre eux, ce à quoi il parviendra effectivement seize mois plus tard. Lorsqu'on février 1985, il voudra prendre des mesures plus sévères a l'égard des Frères, il les accusera d'exploiter la religion à des fins politiques.

41 Les mesures d'islamisation étaient en fait essentiellement une tentative désespérée pour sauver un régime déjà chancelant. Cette démarche n'a cependant servi qu'à semer la discorde et à aggraver les problèmes. Le dommage causé au pays était presque irréparable. Au sud du Soudan, la résistance armée, reconstituée dès le début de 1963, se mua, sous forme de violents combats, en une lutte à très grande échelle.

42 Au nord, le mécontentement et la résistance au gouvernement arbitraire de Nimeyri commencent à galvaniser presque toutes les couches de la société soudanaise. Après le coup inattendu contre les Frères musulmans, le régime de Nimeyri se retrouve complètement isolé. Vers la fin mars 1985, des manifestations et des émeutes d'une violence croissante éclatent à Khartoum et dans les grandes villes. L'Alliance nationale al-tajammu' al-watani, formée de membres de syndicats, de groupes professionnels et $\mathrm{d}$ 'hommes politiques, naît avec les premières vagues de mécontentement populaire en vue d'organiser et de mobiliser le mouvement d'opposition dans des circonstances et avec des moyens qui rappellent de manière frappante la révolution d'octobre 1964. 
43 Mars-avril 1985 : le soulèvement populaire renverse le régime de Nimeyri. La décision prise par le commandement de l'armée de soutenir ce soulèvement plutôt que de l'étouffer joue un rôle primordial. Un gouvernement de transition, composé d'un conseil militaire et d'un cabinet civil (nommé par l'Alliance nationale) est constitué pour préparer, dans un délai d'un an, les élections et la reprise du pouvoir par un gouvernement civil. Le MLPS rejette l'invitation à participer à la nouvelle formation, le commandement militaire qui a dirigé la lutte contre l'ALPS dans le sud appartenant à l'ancien régime et devant, de ce fait, être discrédité.

44 Le gouvernement traditionaliste ne réussit pas à convaincre le MLPS de se joindre au processus politique et il n'est pas disposé à utiliser son mandat pour effectuer les changements drastiques que nécessiterait un nouveau démarrage. Paralysé par son caractère provisoire, le gouvernement se trouve pris dans le feu croisé d'intérêts inconciliables. Il va alors jouer au plus sûr en essayant de gagner du temps. Les élections ont eu lieu comme prévu (sauf dans la majorité des régions du sud aux mains de l'ALPS) et le pouvoir passe au gouvernement de coalition formé des deux principaux partis traditionalistes, al-Umma et le PUD, ainsi que leurs jeunes partenaires des partis politiques du Sud implantés à Khartoum ${ }^{20}$. Sadiq al-Mahdi, chef du Parti al-Umma, est nommé Premier ministre, poste qu'il avait déjà occupé en 1966-1967.

Son leadership va s'avérer inefficace. Les différentes coalitions qui gouverneront sous sa présidence ne vont pratiquement rien réaliser et son administration se révélera, comme le signale un auteur, "d'une indécision atteignant à la paralysie ": "Cette paralysie ne peut être attribuée aux seuls défauts personnels de Sadiq al-Mahdi mais à la nature même d'un gouvernement de coalition et au fait que les solutions à apporter aux différents problèmes pouvaient être contradictoires et s'exclure mutuellement. Le pas à faire pour parvenir à un compromis sur la .question de la guerre civile aurait pu nuire à la coalition. Or, la relance de l'économie dépendait de la possibilité d'obtenir une aide extérieure, et cette, dernière était perçue comme ayant des implications politiques pouvant menacer l'équilibre des forces auquel on était parvenu après les élections. ${ }^{21}$

Les événements qui se sont déroulés au Soudan à la fin des années 80 laissent un profond sentiment de déjà vu. En effet, la ressemblance est frappante entre les développements qui ont suivi la révolution d'octobre 1964, d'une part, et ceux qui se sont produits à la suite des émeutes de mars-avril 1985, d'autre part. La guerre civile au sud du Soudan constituait l'arrière-plan et le dénouement des événements des années 60. De même, la guerre civile, plus ou moins intense, constitue le plus grand défi auquel le pays se trouve confronté depuis le début de 1985. Le malaise s'insinuant et paralysant le corps politique à la fin des années 80 ressemble étrangement à la détresse qui avait tourmenté le pays à la fin des années 60 . La conjoncture économique défaillante qu'avait entraînée une guerre civile coûteuse ainsi que la corruption et la mauvaise administration mettaient le pays dans une situation encore plus catastrophique que lors de la crise des années 60. Le plus surprenant, c'est que le débat des années 80 sur la question controversée de l'islamisation reproduisait exactement les propos des années 60 , comme s'il s'était agi de débats enregistrés que l'on réécoutait en augmentant le volume. C'était comme si, par vengeance, l'histoire se répétait.

47 Et comme pour compléter ce triste scénario, l'échec du processus de démocratisation aboutit, en juin 1989 comme en mai 1969, à une reprise du pouvoir par les militaires.

Islam contre sécularisme 
Après 1985, on assiste à une diminution de l'influence des groupes séculiers au nord, alors que les Frères musulmans, officiellement rebaptisés Front national islamique (FNI) pour se distinguer d'une faction dissidente, parviennent, bien davantage que dans les années 60 , à mettre l'islamisation sur le devant de la scène politique. Or, la polarisation va à rencontre du traditionnel partage nord-sud, et le MLPS a posé comme préalable à toute négociation de paix l'abrogation des lois de septembre 1983. De leur côté, reconnaissant que ces lois constituaient un obstacle à la paix, l'Alliance des syndicats, branche de l'Alliance nationale, réclamait, ainsi que d'autres forces séculières du Nord, leur abolition. Quant aux deux partis traditionalistes, al-Umma et le PUD, ils avaient, pour leur part, déjà dénoncé ces lois comme dénaturant l'islam véritable. Cependant, tant en raison de leurs divergences quant à l'importance de la révision à y apporter, que de leur affiliation à des groupes différents et de la violente opposition du FNI, qui voulait imposer des lois islamiques encore plus rigoureuses, ces deux partis ont été incapables d'obtenir l'annulation ou même le remplacement de ces, lois. A plusieurs reprises, le FNI a menacé de lancer le jihâd (guerre sainte) pour empêcher l'abrogation des lois islamiques de 1983.

Des trois grands partis, le FNI est certainement celui qui a fait preuve de l'islamisme le plus militant et de la motivation la plus forte. Cette forte motivation et la présence active de ses partisans dans les régions urbaines ont permis au parti d'exercer une influence plus grande que ne l'aurait laissé penser sa force politique réelle. Dans un contexte aussi désorganisé et imprévisible que la scène politique de Khartoum, le FNI, en répandant ses opinions politiques et en discréditant, voire en déformant celles de ses adversaires, est apparu comme le parti le mieux organisé et le plus efficace.

Dans sa campagne, le FNI a surtout mis l'accent sur le fait que la jurisprudence islamique était «l'expression de la majorité démocratique » et la source générale du droit. Il a également prôné l'adoption d'un système fédéral. Aucune mention n'a été faite sur le moyen par lequel cette « majorité démocratique » avait été atteinte, à moins qu'on la suppose identique à la "majorité musulmane ». Or, le caractère monolithique de cette «majorité » et son unanimité quant aux vues islamistes n'est qu'une simple présomption.

51 En fait, lorsque l'aile militaire du FNI a monté le coup d'État de 1989, le nouveau gouvernement s'est empressé d'instaurer une constitution islamique et un système fédéral de façade contrôlé en réalité par le centre. L'opposition à ce gouvernement du $\mathrm{FNI}^{22}$ était constituée des partis traditionnels, des syndicats et des groupes séculiers du Nord. Cette opposition représente sans aucun doute la 'majorité » de la population musulmane.

52 L'autre élément, et peut-être le plus significatif, de l'opposition islamisme/laïcisme, c'est le MLPS/ALPS qui, par la nature même de son approche politico-militaire des problèmes de gouvernement au Soudan, est devenu un facteur essentiel dans la manière de les résoudre. Les objectifs de ce mouvement tels qu'ils figurent dans le manifeste de 1983 sont les suivants : libération de tout le territoire soudanais ; unité de son peuple et préservation de l'intégrité de son territoire ; établissement d'un nouveau Soudan démocrate et socialiste où régneraient l'égalité, la justice économique et sociale et le respect des droits de l'homme ; résolution des problèmes nationaux et religieux de manière à satisfaire tous les Soudanais, ceci dans un contexte démocratique et laïc; le mouvement s'engage également à réaliser la restructuration radicale du pouvoir $d u$ 
"gouvernement centrai » et à "mettre un terme aux conditions et à la politique qui ont conduit à un développement très inégal du Soudan ${ }^{23}$.

Le MLPS/ALPS a surtout mis l'accent sur l'aspect national. Pour ce parti, la convention d'Addis Abeba est un échec parce qu'elle n'apporte qu'une solution partielle au problème du Sud. «Faisaient défaut, dans cette convention, les mécanismes d'équilibre et de contrôle, au sens où elle concernait une région qui s'opposait à une autre, s'identifiant avec le gouvernement central. L'idée régionale aurait dû concerner l'ensemble du pays dans la mesure où certaines régions du Nord sont aussi sousdéveloppées que le Sud. C'est la population des différentes localités qui devrait exercer le pouvoir. $»^{24}$

Cependant, les prétentions du MLPS à être un mouvement "nationaliste " sont contestables dans la mesure où, même dans le Sud, la coalition MLPS/ALPS ne représente qu'une seule tribu, celle des Dinka. En fait, les gouvernements successifs ont tenté de tirer parti des rivalités nilotiques entre Dinka et Nuer, et des différends entre Equatoriaux et Dinka. Les milices du Sud, rivales des Dinka, notamment les Anya Nya II, ont été entraînées et armées pour combattre aux côtés de l'armée soudanaise comme " forces alliées ». S'il est vrai que le MLPS/ALPS est essentiellement basé dans les tribus Dinka, il a néanmoins réussi à se faire des partisans parmi les autres tribus du Sud et même du Nord. Les opérations militaires de l'ALPS au sud du Kordofan - ouest du Soudan - montrent bien que ce mouvement a su gagner l'opinion à sa cause et obtenir, de la population de ces régions, un éventuel soutien actif.

Toutefois, lorsqu'on août 1991 deux dirigeants du parti, Lam Akol et Riek Machar, de Nasir (Haut-Nil), tentent d'écarter John Garang de la direction du mouvement, de graves divisions surgissent au sein de l'organisation. Bien que les autres membres de la direction du MLPS soient restés fidèles à Garang, la scission va causer d'importants dégâts politiques et militaires. Sur le plan politique, l'orientation "nationale » est sapée par le groupe de Nasir, qui réclame une sécession "provisoire» du Sud sous prétexte que l'on épuise ses ressources à vouloir poursuivre un objectif inaccessible, celui de la libération de tout le Soudan. Le gouvernement militaire du FNI exploite aussitôt ces divisions et conclut un accord de cessez-le-feu avec le groupe Nasir. Lors de la principale offensive du gouvernement qui réussit, au printemps 1992, à reprendre certaines villes du Sud, le MLPS accuse les troupes de Nasir, sous le commandement de Akol et de Machar, de collaborer avec l'armée soudanaise. L'ALPS, ayant perdu ses bases à la suite de la chute, en Ethiopie, du régime de Mengistu à la fin de 1991, se trouve militairement affaiblie.

Entre-temps, le Nord semble reculer vers le passé. Ceux qui ont pris le pouvoir à la suite du renversement de Nimeyri sont ces mêmes dirigeants issus des confréries traditionalistes qui ont gouverné le pays durant le bref intervalle démocratique, ceuxlà mêmes qui ont mis fin à chacune des phases démocratiques. L'actuel régime militaire cherche à remettre au goût du jour quelques-unes des pratiques les plus scandaleuses de l'époque de Nimeyri. Tout se passe comme si le Nord n'avait tiré aucune leçon de l'histoire. D'une certaine façon, le système politique du Soudan est devenu plus « tribal » sur le plan idéologique et plus conservateur sur le plan politique. Le Soudan était enfin - même si c'était bien tardivement - sur la voie d'une résolution pacifique du problème chronique de la guerre civile, lorsque s'est produit de nouveau le jeu de bascule amenant au pouvoir un gouvernement militaire apparemment décidé à résoudre le problème militairement ou, du moins, à établir une paix conforme aux 
exigences des islamistes. En fait, il est fort probable que le coup d'État ait été monté dans l'intention de faire échouer le processus de paix.

Le Soudan est revenu à la case départ, comme s'il était condamné par une force malveillante à souffrir, une fois de plus, l'agonie et les frustrations de Sisyphe. Cependant, le vrai défi n'est pas tant de rétablir un régime démocratique que de rompre le cycle civil/militaire en s'assurant que le choix démocratique est viable.

Conclusion

59 Dans le contexte soudanais, la confrontation entre institutions occidentales et structures locales ne reflète pas nécessairement le type de dichotomie tradition/ modernité qui existe implicitement dans le modèle occidental de modernisation. La démocratie parlementaire calquée sur le modèle de Westminster a été quasiment le seul moyen de domination utilisé par les dirigeants traditionalistes. Le gouvernement militaire (supposé modernisateur) qui a régulièrement interrompu le système quasi parlementaire n'était pas plus moderniste que les gouvernements civils qu'il a si souvent supplantés en recourant à la force. Dans certains cas, il a même fait preuve de plus de conservatisme. En fait, les traditionalistes sont aussi désunis que les modernistes et la querelle qui les oppose n'est pas simplement une querelle entre anciens et modernes. Les extrêmes radicaux du spectre politique, au nord du Soudan, sont les laïcs et la gauche, d'une part, et les fondamentalistes, d'autre part. Les deux comptent, à des degrés différents, sur le soutien des «lettrés» (intellectuels, syndicalistes, officiers de l'armée, fonctionnaires, étudiants). Les partis traditionalistes évitent de se prononcer de manière aussi radicale et avec autant de militantisme que les fondamentalistes, mais ils sont néanmoins attachés aux valeurs islamiques sans pour cela être dépourvus d'une, volonté et de moyens politiques de modernisation. L'intervention des militaires a le plus souvent été menée contre les partis traditionalistes (même lorsqu'elle avait été organisée à leur instigation ou avec leur complicité), et s'ils ont accepté, après hésitation, de soutenir l'un ou l'autre groupe extrémiste, c'est dans le seul but, plus tard, de se retourner contre lui.

La question du Sud constitue un facteur déterminant dans les cycles civils/militaires. Le passage de l'un à l'autre a été lié à-ou provoqué par - la situation dans le Sud. Absorbés par les problèmes de politique factionnelle, les partis traditionalistes ont accordé une attention toute particulière au dénouement final. Il en a été de même du gouvernement militaire, en dépit de la convention d'Addis-Abeba de Nimeyri.

61 La situation politique se complique avec l'intrusion du facteur islamiste qui vient s'ajouter aux causes de division déjà existantes. L'apparition du MLPS comme mouvement idéologique "nationaliste » basé dans le sud remplace la traditionnelle scission Nord-Sud, mais avec, en plus, une -confrontation islamistes/laïcs. Le dilemme devant lequel le MLPS place les partis traditionalistes, le FNI et le parti militaire, ne se limite pas aux moyens militaires croissants dont dispose l'ALPS, quoique ces moyens aient rendu le Sud incontrôlable et la guerre civile elle-même pratiquement impossible à gagner. Le vrai dilemme se situe au niveau du refus catégorique du MLPS d'être considéré et traité comme un mouvement séparatiste. La division au sein du MLPS, plutôt que d'atténuer ce message, ne fait que le renforcer. Cependant, le fait de refuser de prendre en considération la revendication nationale, ou même, de manière plus simpliste, d'essayer de proposer une alternative entre "l'islamisation» et la «scission» du Nord, ne fera qu'aggraver le conflit et, par conséquent, retarder le 
développement économique et social du pays, peut-être même entraîner son morcellement.

Le régime militaire du FNI, sous le commandement du général al-Bachir, a commencé là où, dans un contexte moins contraignant, le régime Abboud avait échoué il y a plus d'un quart de siècle. A plus d'un égard, le présent reproduit le passé de manière alarmante. La tâche apparemment interminable qui permettrait de 'remonter la pente " a très peu de chances d'aboutir mais doit néanmoins être accomplie si le Soudan veut se sauver de lui-même.

Camus termine sur ces mots son Mythe de Sisyphe: "On doit imaginer Sisyphe heureux. » Pour l'instant, il est difficile d'en dire autant du Soudan...

\section{NOTES}

1. Scruton R., A Dictionary of Political Thought, The Macmillan Press, London, 1982, p. 468.

2. Idem. pp. 468-469.

3. Selon Scruton, « les causes du comportement individuel sont immanentes, c'est-àdire qu'elles existent non pas sous (orme de doctrines explicites, mais en tant que perception de la validité d'un acte au cours de son exécution et en tant que révélateur d'autres motivations dont renonciation est impossible ou, peut-être, non souhaitée. » Ibid., p. 468.

4. Apter D. E., The Politics of Modernization (University of Chicago Press, Chicago and London, 1985), pp. 3-4. Selon Apter, le développement, la modernisation et l'industrialisation sont fonction de la « diminution des généralités conceptuelles ». 5. Lerner D., The Passing of Traditional Society; Modernizing the Middle East. (The Free Press, New-York. 4th édition, 1985), pp. 46-47. Pour Lerner, le processus séculier de changement social par lequel s'est opérée la modernisation de l'Occident est totalement anachronique par rapport au problème actuel de transition du Moyen-Orient. Du reste, l'adoption du modèle occidental nous est quasi imposée par les postulats et les objectifs déclarés des porte-parole du Moyen-Orient », p. 46.

6. Lerner, op.cit., p. 69.

7. Bechtold P. K., Policies in the Sudan; Parliamentary and Military Rule in an Emerging African Nation, Praeger Publishers, New-York. 1976, p. 100. Bechtold cite le cas des fonctionnaires instruits qui, à cause de l'hétérogénéité de la société soudanaise, tirent leur force de leur affiliation à des associations ethniques.

8. Bernstein H., Modernization Theory and the Sociological Study of Development'. The Journal of Development Studies, vol. 7, n 2. January 1981, p. 146.

9. Voir Hamid M.B, «Perceptions, Preference, and Policy: an Afro-Arab Perspective of Anti-Americanism » in Alvin Z. Rubenstein and Donald E. Sith (eds.) Anti-Americanism in the Third World: Implications for US Foreign Policy, Praeger, New-York, p. 88.

10. Anderson R. et al., Politics and Change in the Middle East. Prentice-Hall Inc., NewJersey, 1987, p. 3. 
11. Hamid M.B, « Devolution and the Problems of National Integration: The Case of the Sudan » in Mom K.N. Arou and B. Youngo-Bure (eds.). North-South Relations in the Sudan since the Addis Abeba Agreement, Institute of African and Asian Studies, University of Khartoum, 1988, p.130.

12. Warburg G, Islam, Nationalism and Communism in a traditional Society: The Case of Sudan, Frank Cass, London 1978, p. 149.

13. Ibid., p.150.

14. Woodward P., " Islam and Politics ", in M. Abd-al-Rahman, R. Badal, A. Hardalo and P. Woodward (eds.), Sudan since Independance : Studies of the Political Development since 1956, Gower, London, 1986, p. 3.

15. Hamid M.B, op. cit, p.127.

16. Niblock T., Class and Power in Sudan: The Dynamics of Sudanese Politics. 1898-1985. Mac Millan Press, London. 1987, p. 219.

17. « Les politiques des partis de l'après-révolution d'octobre étaient antagonistes à l'extrême. La scène politique a vu s'opposer les partis traditionalistes, Umma et PUN, entre les différentes factions au sein de ces partis, comme celle qui a opposé Sadiq alMahdi à son oncle Imam al-Mahdi au sein du Parti Ansar, ou Isma'îl al-Azhari au PDP au sein du Parti Khatmiyya, ou encore les communistes et les Frères musulmans, dont les projets étaient incompatibles et dont les idéologies s'excluaient. » Khalid Mansour, Nimeyri and the Revolution of Dix May, KPI, London, 1985, p. 6.

18. Pour un bilan de la politique étrangère du régime Nimeyri, voir Hamid M.B, «The Finlandization of Sudan's Foreign Policy ", Journal of Arab Affairs (Fresno), vol. 2, Spring 1983.

19. Pour une étude détaillée de la politique de réconciliation nationale au Soudan, voir, du même auteur, The Politics of National Reconciliation in the Sudan : The Nimeyri Regime and the National Front Opposition (Center for Contemporary Arab Studies, Georgetown University. 1984).

20. Le Parti de l'Union Démocratique est né de la scission du PUN et du PDP.

21. Ottaway M.. « Post-Nimeyri Sudan; One Year on », Third World Quarterly (London), vol. $9, \mathrm{n}^{\circ} 3$.

22. "Gouvernement du FNI » : l'expression est de l'auteur, le rôle du FNI au sein de l'actuel gouvernement soudanais n'étant pas officiel ( $n d l$ ).

23. Khalid M. (ed.), John Garang Speaks. London. LIPI limited, 1987, p. 26.

24. Interview de Lam Akol Ajawin du Haut commandement militaire de l'ALPS, par Africa Report, reproduit dans le Sudan Times (Khartoum), 30 juin 1987.

\section{INDEX}

Mots-clés : Soudan 
AUTEUR

MOHAMMED BESHIR HAMID

Université de Khartoum 